\title{
Successful desensitization therapy involving fluoroquinolone for the treatment of a solitary tuberculoma: A case report and literature review
}

\author{
HIDEHIRO WATANABE ${ }^{1,2}$, TOMONORI URUMA ${ }^{1,2}$, IKUO SEITA ${ }^{1}$, YUSHI CHIKASAWA ${ }^{1}$, RYOTA KIKUCHI ${ }^{2}$, \\ MASAYUKI ITOH $^{2}$, KAZUTETSU AOSHIBA ${ }^{2}$, HIROYUKI NAKAMURA $^{2}$ and TSUYOSHI OISHI ${ }^{1}$ \\ Departments of ${ }^{1}$ Infection Control and Infectious Disease, and ${ }^{2}$ Respiratory Medicine, \\ Ibaraki Medical Center, Tokyo Medical University, Inashiki, Ibaraki 300-0395, Japan
}

Received July 17, 2015; Accepted April 19, 2016

DOI: $10.3892 / \mathrm{mco} .2016 .881$

\begin{abstract}
The patient was a 31-year-old female with no previous health problems; however, during a health checkup in 2013 , a nodule $(2.5 \mathrm{~cm}$ in diameter) was identified in the S10 area of the left lung. No clinical symptoms were apparent. Positron emission tomography/computed tomography revealed an accumulation in the same region. The patient was suspected of having lung cancer, and video-assisted thoracoscopic surgery was performed. A histopathological examination of the resected specimen revealed epithelioid granulomas accompanied by caseous necrosis in the lesion. The culture was positive for Mycobacterium tuberculosis, which led to the final diagnosis of tuberculoma. Initially, the patient underwent anti-M.tuberculosis treatment [isoniazid $(\mathrm{INH})+$ rifampicin $(\mathrm{RFP})+$ ethambutol $(\mathrm{EB})+$ pyrazinamide (PZA)]. However, two weeks later, the development of epatic dysfunction necessitated suspension of the medication. Treatment was resumed following improvement of the hepatic function. However, this relapsed two weeks later, resulting in discontinuation of the treatment. The patient was negative for each of the four drugs in the drug-induced lymphocyte stimulation test (DLST), and drug-induced hepatotoxicity (DIH) attributable to the anti-tuberculous drugs that were administered. Therefore, desensitization therapy was initiated. $\mathrm{EB}+\mathrm{PZA}$ were changed to levofloxacin (LVFX) at an initial dose of $250 \mathrm{mg} /$ day (dose level increased to the maintenance dose). Subsequently, desensitization therapy with RFP and INH was applied in accordance with the Japanese Society for Tuberculosis protocol. After each drug dose level reached the
\end{abstract}

Correspondence to: Dr Hidehiro Watanabe, Department of Infection Control and Infectious Disease, Ibaraki Medical Center, Tokyo Medical University, 3-20-1 Chuo, Ami, Inashiki, Ibaraki 300-0395, Japan

E-mail: hw-nabe4@tokyo-med.ac.jp

Key words: tuberculoma, drug-induced hepatotoxicity, desensitization therapy, fluoroquinolone maintenance dose level, the therapy was completed following administration of the drugs for the recommended duration of 6 months. There were no signs of relapse 6 months following completion of the therapy. Therefore, the patient responded well to the substitute therapy with LVFX and desensitization therapy, and the present case report provided information regarding the treatment of tuberculoma.

\section{Introduction}

Tuberculoma is considered a benign disease, and accounts for $5-24 \%$ of all solitary pulmonary nodules (SPNs) (1). Of all cases of tuberculoma, $77.3 \%$ are asymptomatic and only detected during routine health checkups. Tuberculoma assumes the form of SPN in $77-85 \%$ of all cases, and two or more nodules or satellite lesions are present in $15-22 \%$ of total cases. The nodules are commonly located in the upper lobes of the lungs and frequently measure $2-5 \mathrm{~cm}$ in diameter $(2,3)$.

The likelihood of a malignant in the SPN is known to increase with the size of it. For SPNs measuring $<5 \mathrm{~mm}$ in diameter, the probability of malignancy is $0-1 \%$, whereas with an increase in the diameter to $11-12 \mathrm{~mm}$, the probability of the tumor becoming malignant increases to 33-64\%. With a further increase in the diameter to $20 \mathrm{~mm}$, the likelihood of malignancy increases to $64-82 \%$ (4). The efficacy of positron emission tomography (PET) in the management of cancer has been reported previously $(4,5)$. However, a distinction between tuberculoma and lung cancer is extremely difficult to make, even with the use of PET (5).

Desensitization therapy for the treatment of tuberculoma has rarely been reported previously. The present study reports a case of desensitization therapy in the treatment of a patient with solitary pulmonary tuberculoma, and provides reference to the current literature. The solitary pulmonary tuberculoma was diagnosed by video-assisted thoracoscopic surgery (VATS) and treatment with antituberculous drugs resulted in drug-induced hepatotoxicity (DIH). Subsequently, desensitization therapy with quinolones and other therapeutic agents proved effective in treating the patient's tuberculoma. 


\section{Case report}

The patient was a non-smoking 31-year-old female who does not consume alcohol and had no history of health problems. However, during a routine health checkup on November 26th 2013, an intramural nodule $(2.5 \mathrm{~cm}$ in diameter) in the $\mathrm{S} 10$ area of the left lung was identified. The patient was asymptomatic and PET-computed tomography (CT) revealed accumulation (standardized uptake value of 4.5) in the lesion (Figs. 1A and B). Therefore, the patient was suspected to have lung cancer and VATS was performed on December 10th 2013. Histopathological examination of the resected specimen revealed the presence of epithelioid granulomas, accompanied by caseous necrosis in the lesion (Fig. 1C). The tissue specimen was negative on Ziehl-Neelsen staining, however, culture for Mycobacterium tuberculosis was positive (M. tuberculosis antigen-positive), which led to a final diagnosis of tuberculoma. Anti-M. tuberculosis treatment [isoniazid (INH) + rifampicin (RFP) + ethambutol (EB) + pyrazinamide (PZA)] commenced on January 13th 2014; however, the patient developed hepatic dysfunction [aspartate transaminase/alanine transaminase (AST/ALT): 506/867] on February 1st 2014 (Table I), which necessitated the suspension of the treatment. The medications were resumed $(\mathrm{EB}+\mathrm{PZA})$ following improvement of the hepatic function; however, the hepatic dysfunction (AST/ALT: 455/928) relapsed on March 12th 2014 (two weeks following the resumption) and the treatment was terminated. During this period, tests for hepatitis virus markers and human immunodeficiency virus were negative. Based on the hypothesis that the patient may have an allergy to antituberculous drugs, the drug-induced lymphocyte stimulation test (DLST) was performed for the four antituberculous drugs; however, the data were within the criterion range for each of the drugs. Two months elapsed before the patient's hepatic function was recovered. Although the patient tested negative for DLST, a diagnosis of drug-induced hepatotoxicity (DIH) due to the antituberculous drugs was made on the basis of the clinical course. Standard treatment with antituberculous drugs was considered to be associated with risks; therefore, reductive-sensitizing therapy with antituberculous drugs was performed. On May 12th 2014, the treatment with EB + PZA was changed to levofloxacin (LVFX) at the initial dose of $250 \mathrm{mg} /$ day. This dose level was increased to the maintenance dose level of $500 \mathrm{mg}$ /day following confirmation of the absence of deteriorating hepatic function one week later. Subsequently, on May 19th 2014, desensitization therapy was initiated in accordance with the protocol of the Japanese Society for Tuberculosis (JST) (6). Firstly, RFP (25 mg/day) was used and its dose level was gradually increased at intervals of 3 days to the maintenance dose level (450 mg/day). Following confirmation of a lack of deteriorating hepatic function, INH was added on June 16th 2014 at $25 \mathrm{mg}$ /day, and the dose level increased at intervals of 3 days to the maintenance dose level (300 mg/day). Subsequent to the dose level of each drug reaching the maintenance dose level (LVFX, $500 \mathrm{mg} /$ day; RFP, $450 \mathrm{mg} /$ day; and $\mathrm{INH}, 300 \mathrm{mg} /$ day), the therapy was continued for an additional 6 months and completed on January 7th 2015. During this period, there were no cases of hepatic function deterioration or any other adverse events. During the subsequent follow-up, no signs of relapse were detected.

\section{Discussion}

The reported incidence of tuberculosis in 2014 was $16.1 / 100,000$ of the population in Japan, 3.1 in USA, 4.7 in Canada, 4.9 in Germany and 5.7 in Australia, with the incidence being significantly higher in Japan compared with in the Western countries (7). Tuberculoma is thought to develop in $6-9 \%$ of all patients with post-primary tuberculosis (2), and when it assumes the form of an SPN, its distinction from cancer is difficult. Therefore, VATS-based resection and histological diagnosis are recommended for the differential diagnosis in such cases $(5,8,9)$. After INH became available for clinical use in the 1960s, numerous studies on anti-tuberculous drugs and DIH were performed in that decade. According to reports after 1990, the year in which the three-drug regimen of INH + RFP + PZA was established as a standard therapy, DIH (defined as a serum AST level of $\geq 100 \mathrm{U} / \mathrm{ml}$ and/or serum total bilirubin level of $\geq 2.5 \mathrm{mg} / \mathrm{dl}$ ) occurred at an incidence of $2.4 \%$ in cases treated with INH + RFP + PZA for 2 months and INH + RFP for the subsequent 4 months. The reported incidence following 9 months of treatment with INH + RFP + EB was $3.6 \%$. The incidence of DIH has been demonstrated not to vary depending on the presence/absence of PZA in the treatment regimen (10). According to reports after 2000, the incidence of DIH following tuberculosis treatment has become higher, with inter-ethnic differences in the frequency (11-13). Comparison of the DIH incidence between two major standard therapies, i.e., the British Thoracic Society (BTS) and American Thoracic Society (ATS) reintroduction guidelines for antituberculous therapy, revealed no difference in the incidence (BTS, 9.8-16\%; ATS, 11.1-18\%) (14). The possible risk factors for DIH include age ( $>35$ years old and children), gender (female), extent of tuberculosis (spread of the disease beyond the lungs), malnutrition (serum albumin, $<3.5 \mathrm{mg} / \mathrm{dL}$ ), alcohol consumption, presence of hepatitis, drug dose level and genetic polymorphism (three major enzymes involved in INH degradation are $\mathrm{N}$-acetyltransferase 2, cytochrome P450 2E1, and glutathione S-transferase) (15). However, certain previous studies have reported the absence of any association of NIH with the body mass index or serum albumin level $(14,16)$.

Among the previous reports providing collective data on the cases of tuberculoma, Lee et al (2) reported the results of treatment in 45 cases of tuberculoma, of which 24 (53.3\%) were asymptomatic and detected during a routine health checkup. Of the 45 patients, $7(15.6 \%)$ had a previous history of tuberculosis and $13(28.9 \%)$ had a history of diabetes mellitus. The treatment consisted of INH, RFP, EB and PZA in 38 patients, INH, RFP and EB in six patients, and EB, streptomycin, cycloserine and LVFX in one patient. In six (13.3\%) patients, the first-line drugs were switched to second-line drugs due to the appearance of DIH; however, there was no description in the report of desensitization therapy. According to a report by Hsu et al (3) of 53 tuberculoma cases, $41(77.4 \%)$ were asymptomatic and detected during a routine health check-up, while 12 (22.6\%) had respiratory symptoms (including coughing and sputum). Following VTAS, standard antituberculous therapy was administered for 6-12 months; however, there was no description in the report on the occurrence of DIH. Laisaar et al (17) reported the results of 43 cases of tuberculoma, in which 37 patients $(86.0 \%)$ received treatment with first-line drugs 
Table I. Laboratory findings of the liver functions in the clinical course.

2014

2013

\begin{tabular}{|c|c|c|c|c|c|c|c|}
\hline \multirow[b]{2}{*}{ Test } & \multirow[b]{2}{*}{ Unit } & \multirow{2}{*}{$\begin{array}{c}2013 \\
\text { November 26th }\end{array}$} & & \multirow{2}{*}{$\begin{array}{c}2015 \\
\text { January 28th }\end{array}$} \\
\hline & & & February $1 \mathrm{st}$ & February 26th & March 12th & May 12th & \\
\hline T-Bil & $\mathrm{mg} / \mathrm{dl}$ & 0.6 & 0.6 & 0.5 & 1.3 & 0.9 & 0.5 \\
\hline $\mathrm{AST}$ & IU/1 & 14.00 & 506.00 & 38.00 & 455.00 & 12.00 & 12.00 \\
\hline ALT & $\mathrm{IU} / 1$ & 15.00 & 867.00 & 74.00 & 928.00 & 12.00 & 12.00 \\
\hline$\gamma$-GTP & IU/1 & NP & 253.00 & NP & NP & 30.00 & 25.00 \\
\hline LDH & IU/1 & 183.00 & 472.00 & 198.00 & 341.00 & 164.00 & 169.00 \\
\hline ALP & IU/1 & 187.00 & 446.00 & NP & NP & 189.00 & 195.00 \\
\hline $\mathrm{Cr}$ & $\mathrm{mg} / \mathrm{dl}$ & 0.51 & 0.57 & 0.46 & 0.53 & 0.53 & 0.46 \\
\hline CRP & $\mathrm{mg} / \mathrm{dl}$ & NP & 5.34 & 0.06 & 0.26 & 0.07 & 0.05 \\
\hline Plt & $10^{4} / \mu 1$ & 28.8 & 17.8 & 29.6 & 21.1 & 24.5 & 23.8 \\
\hline
\end{tabular}

T-Bil, total bilirubin; AST, aspartate aminotransferase; ALT, alanine transaminase; GTP, guanosine triphosphate; LDH, lactate dehydrogenase; ALP, alkaline phosphatase; Cr, creatine; CRP, creatine phosphate; Plt, platelet; NP, not performed.

A

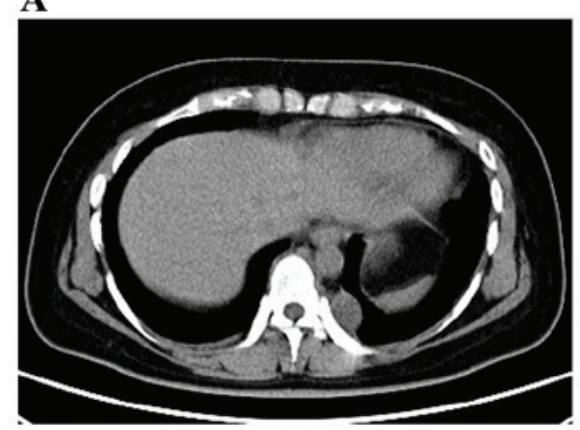

B

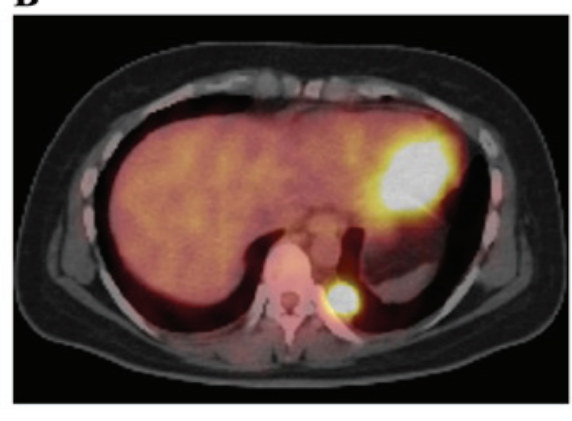

C

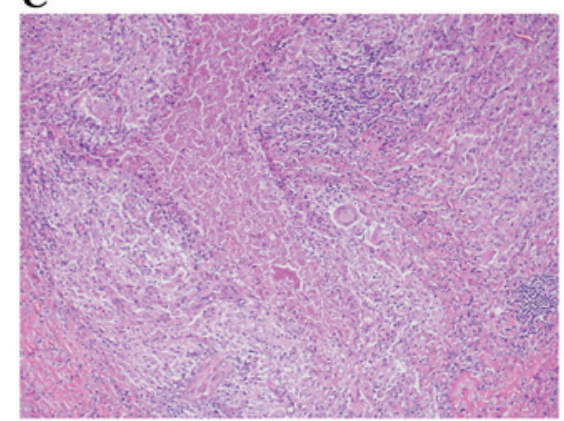

Figure 1. (A) A nodule (2.5 cm in diameter) observed on the pleura in the $\mathrm{S} 10$ area of the left lung by chest CT. (B) The marked accumulation of cancer-like cells was observed in positron emission tomography-CT (maximum standardized uptake value, 4.5). (C) The presence of epithelioid granulomas accompanied by caseous necrosis in the nodule. (magnification, x100) CT, computed tomography.

and $5(11.6 \%)$ received treatment with second-line drugs. During the follow-up period lasting 9 years, no patient relapsed subsequent to the postoperative drug therapy (mean duration, 185 days). With regards to special treatments, Yang et al (18) reported that 54 patients of tuberculoma were treated by direct injection of $0.1 \mathrm{~g} \mathrm{INH}$ and $0.2 \mathrm{~g}$ amikacin into the tuberculoma, which resulted in a marked decrease in the size or disappearance of the tuberculoma in 31 patients (57.4\%) following 10 treatment sessions. The adverse reactions observed were pneumothorax (5 cases, 9.3\%), hemoptysis (4 cases, 7.4\%) and pyrexia (6 cases, 11.1\%); however, there was no report of DIH development.

The diagnosis of DIH during antituberculous drug treatment was based on the finding of elevated serum levels of the hepatic enzymes and the accompanying clinical symptoms. The judgment of 'positive' in the DLST with drugs is not an absolute requirement for the diagnosis of DIH, as the reported DLST-positive rate is only $56 \%$ for RFP and $50 \%$ for INH (6). Furthermore, a previous study reported that the onset of DIH bears no correlation with the blood levels of INH, RFP, EB or PZA (19). Additionally, in the present case, the DLST was negative for INH, RFP, EB and PZA; however, hepatic dysfunction developed following the administration of the second medication, suggesting the strong possibility that DIH was attributed to EB and PZA in the present patient.

Desensitization therapy has been used as one of the methods for the management of DIH, which occurs during antituberculous treatment. The desensitization protocol used differs between Japan and Western countries. Kobashi et al (6) reported the differences as follows: While the initial dose of IHN or RFP starts at $25 \mathrm{mg} /$ day and is gradually increased every 3 days over a period of $>2$ weeks in the desensitization therapy proposed by the JST, in Western countries the dose starts from $0.1 \mathrm{mg}$ every $45 \mathrm{~min}$ and requires only 2 days to complete the desensitization therapy. The previous study additionally reported that the protocol for desensitization therapy used in Western countries for DIH to antituberculous drugs is based on the penicillin desensitization therapy. Furthermore, these investigators reported that the desensitization therapy administered in accordance with the JST protocol had a success rate of $77 \%$ for the case of RFP and $81 \%$ for the case of INH, which is more efficacious compared with the outcome of the therapy applied in accordance with the Western protocol. Another previous study also reported that substitute therapy 
using levofloxacin or moxifloxacin instead of INH and RFP was effective (20). In the present case, LVFX was used initially at half the ordinary dose level when the medication was resumed, and desensitization therapy with RFP and INH was applied in accordance with the JST protocol (6), which resulted in a favorable outcome and no relapse.

In conclusion, the reported incidence of DIH to antituberculous drugs used in the treatment of tuberculoma was $\leq 15 \%$. Following the onset of DIH, the majority of patients continued to receive treatment, although the drugs were frequently switched to second-line drugs. The literature identified no previous studies that utilised the application of desensitization therapy to treat DIH. The present study reports a case of tuberculoma, which required an initial differentiation from lung cancer and was diagnosed on the basis of VATS findings. The patient developed DIH twice following antituberculous drug treatment, and the DIH in this patient appeared to be attributable to EB and PZA. Substitute therapy using LVFX and desensitization therapy with INH and RFP were applied, which yielded a favorable outcome. Therefore, the present study signifies the importance of DIH management with regards to antituberculous drugs.

\section{References}

1. Andreu J, Cáceres J, Pallisa E and Martinez-Rodriguez M: Radiological manifestations of pulmonary tuberculosis. Eur J Radiol 51: 139-149, 2004.

2. Lee HS, Oh JY, Lee JH, Yoo CG, Lee CT, Kim YW, Han SK, Shim YS and Yim JJ: Response of pulmonary tuberculomas to anti-tuberculous treatment. Eur Respir J 23: 452-455, 2004.

3. Hsu KY, Lee HC, Ou CC and Luh SP: Value of video-assisted thoracoscopic surgery in the diagnosis and treatment of pulmonary tuberculoma: 53 cases analysis and review of literature. J Zhejiang Univ Sci B 10: 375-379, 2009.

4. Zhan P, Xie H, Xu C, Hao K, Hou Z, et al: Management strategy of solitary pulmonary nodules. J Thorac Dis 5: 824-829, 2013.

5. Sathekge MM, Maes A, Pottel H, Stoltz A and van de Wiele C: Dual time-point FDG PET-CT for differentiating benign from malignant solitary pulmonary nodules in a TB endemic area. S Afr Med J 100: 598-601, 2010.

6. Japan, Ministry of Health,Labour and Welfare. http://www.mhlw. go.jp/bunya/kenkou/kekkaku-kansenshou03/dl/13. Accessed June 10, 2015.
7. Luh SP and Liu HP: Video-assisted thoracic surgery-the past, present status and the future. J Zhejiang Univ Sci B 7: 118-128, 2006.

8. Totanarungroj K, Chaopotong S and Tongdee T: Distinguishing small primary lung cancer from pulmonary tuberculoma using 64-slices multidetector CT. J Med Assoc Thai 95: 574-582, 2012.

9. Combs DL, O'Brien RJ and Geiter LJ: USPHS Tuberculosis Short-Course Chemotherapy Trial 21: Effectiveness, toxicity and acceptability. The report of final results. Ann Intern Med 112: 397-406, 1990.

10. Tostmann A, Boeree MJ, Aarnoutse RE, de Lange WC, van der Ven AJ and Dekhuijzen R: Antituberculosis drug-induced hepatotoxicity: Concise up-to-date review. J Gastroenterol Hepatol 23: 192-202, 2008.

11. Saukkonen JJ, Cohn DL, Jasmer RM, Schenker S, Jereb JA, Nolan CM, Peloquin CA, Gordin FM, Nunes D, Strader DB, et al; ATS (American Thoracic Society) Hepatotoxicity of Antituberculosis Therapy Subcommittee: An official ATS statement: Hepatotoxicity of antituberculosis therapy. Am J Respir Crit Care Med 174: 935-952, 2006.

12. Ohno M, Yamaguchi I, Yamamoto I, Fukuda T, Yokota S, Maekura R, Ito M, Yamamoto Y, Ogura T, Maeda K, et al: Slow $\mathrm{N}$-acetyltransferase 2 genotype affects the incidence of isoniazid and rifampicin-induced hepatotoxicity. Int J Tuberc Lung Dis 4: 256-261, 2000.

13. Zuberi BF, Zuberi FF, Bader N, Alvi H and Salahuddin J: Comparison of British Thoracic Society and American Thoracic Society reintroduction guidelines for anti-tuberculous therapy induced liver injury. J Pak Med Assoc 64: 896-899, 2014.

14. Devarbhavi H: Antituberculous drug-induced liver injury: Current perspective. Trop Gastroenterol 32: 167-174, 2011.

15. Abbasi MA, Ahmed N, Suleman A, Zaman H, Tariq S, Anwar SA and Khan N: Common risk factors for the development of anti tuberculosis treatment induced hepatotoxicity. J Ayub Med Coll Abbottabad 26: 384-388, 2014

16. Laisaar T, Viiklepp P and Hollo V: Long-term follow-up after thoracoscopic resection of solitary pulmonary tuberculoma. Indian J Tuberc 61: 51-56, 2014.

17. Yang SH, Zhan P, Mao HH, Shi XD and Wang LL: Perfusing chemotherapy by percutaneous lung puncture 'holing' for pulmonary tuberculoma-a ten-year single center experience. J Thorac Dis 5: 466-471, 2013.

18. Kobashi Y, Abe T, Shigeto E, Yano S, Kuraoka T and Oka M: Desensitization therapy for allergic reactions to antituberculous drugs. Intern Med 49: 2297-2301, 2010.

19. Jeong I, Park JS, Cho YJ, Yoon HI, Song J, Lee CT and Lee JH: Drug-induced hepatotoxicity of anti-tuberculosis drugs and their serum levels. J Korean Med Sci 30: 167-172, 2015.

20. Ho CC, Chen YC, Hu FC, Yu CJ, Yang PC and Luh KT: Safety of fluoroquinolone use in patients with hepatotoxicity induced by anti-tuberculosis regimens. Clin Infect Dis 48: 1526-1533, 2009. 